\title{
The effect of mood on word recognition
}

\author{
SCOTT A. SMALL \\ New York University, New York, New York \\ (Joan Gay Snodgrass, Sponsor)
}

\begin{abstract}
There recently has been an upsurge in studies that have shown the influences of emotional mood on a variety of cognitions. Few studies, however, have demonstrated successfully this influence on perception. This study measured tachistoscopic recognition thresholds of two groups of subjects, a depressed-mood-induced group and a neutral group, for words that were either depressively congruent or neutral. The results revealed that recognition thresholds for depressively congruent words were lower for depressively induced subjects than for neutral subjects. These results are discussed in relation to current cognitive theories of emotion.
\end{abstract}

Bower (1981) proposed a theory that incorporates emotional states into the semantic network model of memory (Anderson, 1976; Anderson \& Bower, 1973). Mood states are represented as nodes, the activation of which spreads to other linked nodes, such as perceptual categories, themes, and words that are related to these mood states. Based on this proposed theory, moods should influence several cognitive processes. Indeed, much research has been performed on a variety of cognitive processes and validated this hypothesis (Bargh, 1982; Bower, 1981; Bower, Monteiro, \& Gilligan, 1978; Ellis, Thomas, \& Rodriguez, 1984). For some reason, however, negligible research has been done on the effect of mood on an elementary cognitive process, such as perception. There is a large body of literature that focuses on motivational influences on perception, as reviewed by Erdelyi (1974), but most of these studies have used stimuli (e.g., taboo words) that were not necessarily related to the subject's mood. Most of the other studies in the literature, following Erdelyi's review, either have not tested the functional relationship between mood and perception (e.g., Zajonc, 1980), or have not been "true" experiments, in the sense that the moods of the subjects were not experimentally manipulated (e.g., Bargh, 1982).

The experiments that tested the relation between mood and perception were performed by Gerrig and Bower (1982). They measured recognition threshold for words that were mood congruent, mood incongruent, and neutral, by subjects that were hypnotically induced to be happy or angry. Their results, in contradiction to their hypothesis, yielded no difference in recognition thresholds between mood-congruent words and mood-incongruent or neutral words. In an attempt to salvage their hypothesis-namely, that mood influences cognitive processes-they conclude by stating that mood may in-

\footnotetext{
This research was performed as a final project for a laboratory course in human cognition. I thank Professor Joan Gay Snodgrass for her valuable comments on an earlier draft of this paper. Requests for reprints should be sent to Scott A. Small, Department of Psychology, New York University, 6 Washington Place, 4th Floor, New York, NY 10003.
}

fluence only the retrieval speed of the affective qualities of words but may not influence "lower level" cognitive processes, such as recognition thresholds, that are automated skills.

The purpose of this paper is to show that lower level cognitions do reflect the influence of mood, and that the reason for Gerrig and Bower's (1982) null finding is due to three main flaws in their method: (1) In creating three sets of stimuli- 5 synonyms for happy, 5 synonyms for angry, and 20 neutral words-Gerrig and Bower matched across these sets for word frequency, length in letters, and number of syllables. It is my opinion that these sets are not sufficiently matched, in that two sets of words, happy and angry, were categorically related within each of their respective sets, while the third set, neutral words, did not belong to one single category. Thus, when all the stimuli were presented to the subjects, regardless of mood type, the subjects became sensitized to happy and angry words by virtue of the repetitiveness of words belonging to the same category; this sensitization did not occur for neutral words because they belonged to a random variety of categories. (2) The happy and angry words were not chosen from a standardized list of words that have been shown to be associated to happy and angry moods. (3) Any mood might influence the perception of any word with affective qualities, independent of the congruence between the specific mood of the subject and the specific mood of the word. For example, a subject induced to be happy might be sensitive, though perhaps in different degrees, to both happy and angry words. Accordingly, the way to functionally separate groups of subjects is by mood inducing one group and maintaining the neutrality of the other.

In an attempt to support the hypothesis that mood does influence perception, this experiment was performed with a refined methodology: (1) The sets of stimuli matched according to number of letters and frequency of occurrence in English and belonged to two mutually exclusive categories. (2) The words were chosen from standardized lists of words that are associated to the mood types of the subjects. (3) One group of subjects had a mood of depres- 
sion induced, and the mood of a second group was controlled for neutrality.

\section{METHOD}

\section{Subjects and Design}

The subjects were 10 New York University students enrolled in an introductory psychology course. They participated in the experiment to fulfill the course requirement.

In order to test the influence of mood on perception, the experiment consisted of four experimental conditions (see Table 1): two conditions for mood category, depressive and neutral, and two conditions for word category, depressive and neutral. Thus, this experiment used a $2 \times 2$ mixed design, in which the subjects were crossed with word category and nested within mood category. The dependent variable was tachistoscopic recognition thresholds for words.

\section{Apparatus}

A Scientific Prototype two-channel tachistoscope, Model 800-F, was used to measure individual recognition thresholds.

The stimulus consisted of 20 words that were printed in uppercase on $3 \times 5$ index cards. This list was made up of two equal sets of words in which one set consisted of 10 depressively mood-congruent words (LONELY, GLOOMY, SAD, MEEK, DESPAIR, WORTHLESS, WEARY, LOUSY, INFERIOR, GUILTY), and the other set consisted of 10 neutral words (CLOCK, MIRROR, FOYER, PIANO, STOOL, BOOKCASE, LAMP, COUCH, FIREPLACE, CEILING). These two sets were matched on two dimensions: (1) number of letters-both sets of words had an average of 5.9 letters per word, ranging from 3 to 9 letters, and (2) frequency of occurrence in English-both sets of words had an average of 16 per million, ranging from 3 to 38 (Kučera \& Francis, 1967). The words of each set belonged to mutually exclusive categories. The mood-congruent words were chosen from a standardized list of depression-related words (Dahl \& Stengel, 1978), and the neutral words were chosen from a standardized list of items in a living room (Shapiro \& Palermo, 1970).

One nonword was created, consisting of four letters (CVCV), that was used as a practice stimulus.

\section{Procedure}

Subjects were randomly assigned to the two groups, depressive or neutral (five per group). Subjects in the depressive condition were mood induced by using the technique developed by Velten (1968). This procedure has been successfully employed elsewhere (Hale \& Strickland, 1976; Leight \& Ellis, 1981). With this procedure, subjects read a series of 50 self-referent statements (e.g., "I feel rather sluggish now") that progressively increase in severity in order to induce a depressive mood. Subjects in the neutral condition read Velten's 50 neutral statements. These statements are not self-referent and have no affective qualities (e.g., " $99.1 \%$ of Alaska is owned by the federal government").

After subjects read their assigned mood statements, all of the subjects' recognition thresholds were measured for all of the stimuli. Each subject was initially presented with the practice nonword, to clarify the procedure and to allow the eyes to adjust to the flashes of light. The 20 words were randomized independently for each subject. The words were then presented one by one, at a starting exposure time of $100 \mathrm{msec}$. The exposure time was progressively increased by increments of $10 \mathrm{msec}$ until the subject was able to show recognition of the word by stating what it was. There was no penalty for wrong attempts; therefore the subjects were allowed, though not encouraged, to guess.

Table 1

Mean Recognition Thresholds for Each Experimental Condition (in Milliseconds)

\begin{tabular}{ccc}
\hline & \multicolumn{2}{c}{ Subject Category } \\
\cline { 2 - 3 } Word Category & Depressed & Neutral \\
\hline Depressed & 125 & 142 \\
Neutral & 138 & 141 \\
\hline
\end{tabular}

\section{RESULTS AND DISCUSSION}

The mean tachistoscopic recognition thresholds for each experimental condition are presented in Table 1. It should be noted that the depressed group recognized the word on the first exposure $(100 \mathrm{msec}) 14$ times for the depressive words and 9 times for the neutral words. The neutral group recognized the word on the first exposure 4 times for the depressive words and 3 times for the neutral words. (When the word was recognized on its first presentation, $100 \mathrm{msec}$ was used as the threshold value.)

A $2 \times 2$ analysis of variance was performed on the mean threshold recognition times, in which the first factor was mood category (depressed and neutral), and the second factor was word category (depressed and neutral). There was no significant difference between the subjects that were depressively induced and the subjects that were neutral $(F<1)$. There was a significant difference between stimuli that were depressively congruent and stimuli that were neutral $[F(1,8)=7.68 ; p<.05]$. Most important, there was a significant interaction between these two factors $[F(1,8)=11.12 ; p<.01]$. Depressively induced subjects recognized depressively congruent words at a lower threshold than neutral words, while neutral subjects recognized these two sets of stimuli equally well.

In order to determine the source of the interaction, simple comparisons were performed. First, the mean recognition thresholds of depressive subjects and neutral subjects were compared for the depressively congruent stimuli. This comparison yielded a significant difference between the categories of subjects $[\mathrm{F}(1,8)=34.15$; $p<.001]$. Second, the mean recognition thresholds of depressive and neutral subjects were compared for the neutral stimuli. In accordance with the hypothesis, this comparison yielded no difference between the subject categories $[\mathrm{F}(1,8)=1.28 ; \mathrm{p}>.05]$.

The results offer strong support for this study's general hypothesis that mood-induced subjects will recognize mood-congruent stimuli faster than neutral stimuli. Specifically, neutral subjects recognized all stimuli at equal thresholds, independent of word category, but depressively induced subjects recognized the mood-congruent words at lower thresholds than did neutral subjects.

These results are supportive of and predicted by Bower's theory of emotions (1981), which states that different moods are represented as nodes in the semantic network model (Anderson, 1976; Anderson \& Bower, 1973). In accordance with this model, these mood nodes are linked to a variety of other nodes, including nodes that represent words that are qualitatively related to the mood. Thus, when a person is in a sad mood, there is subthreshold activation of mood-congruent words, such as LONELY. Due to this internal priming, it would take less perceptual excitation of the word LONELY to push it above threshold recognition than it would for a neutral word, such as CLOCK. More specifically, the word LONELY is predicted to have a lower recognition threshold than the word CLOCK, as was found in this experiment. 


\section{REFERENCES}

ANDERSON, J. R. (1976). Language, memory, and thought. Hillsdale, NJ: Erlbaum.

ANDERSON, J. R., \& BowER, G. H. (1973). Human associative memory. Washington, DC: Winston.

BARGH, J. A. (1982). Attention and automaticity in the processing of self-relevant information. Journal of Personality \& Social Psychology, 3, 425-436.

Bower, G. H. (1981). Mood and memory. American Psychologist, 36, 129-148.

Bower, G. H., Monteiro, K. P., \& Gilligan, S. G. (1978). Emotional mood as a context of learning and recall. Journal of Verbal Learning \& Verbal Behavior, 17, 573-585.

DAHL, H., \& STENGEL, B. (1978). Classification of emotional words. Psychoanalysis \& Contemporary Thought, 1, 269-312.

Ellis, H. C., Thomas, R. L., \& RodrigUeZ, I. A. (1984). Emotional mood states and memory: Elaborative encoding, semantic processing, and cognitive effort. Journal of Experimental Psychology: Learning, Memory, \& Cognition, 10, 470-482.

ERdelyi, M. H. (1974). A new look at the new look: Perceptual defense and vigilance. Psychological Review, 81, 1-24.
Gerrig, R. J., \& Bower, G. H. (1982). Emotional influences on word recognition. Bulletin of the Psychonomic Society, 19, 197-200.

Hale, W. D., \& STrickland, B. R. (1976). Induction of mood states and their effect on cognitive and social behavior. Journal of Consulting \& Clinical Psychology, 44, 155.

Kučera, H., \& Francis, W. N. (1967). Computational analysis of presentday American English. Providence, RI: Brown University Press.

LEIGHT, K. A., \& ElLIs, H. C. (1981). Emotional mood states, strategies, and state-dependency in memory. Journal of Verbal Learning \& Verbal Behavior, 20, 251-266.

Shapiro, S. I., \& PAlermo, D. S. (1970). Conceptual organization and class membership: Normative data for representatives of 100 categories. Psychonomic Monograph Supplements, 3, 107-127.

VeLTEN, E. (1968). A laboratory task for induction for mood states. Behavior Research \& Therapy, 6, 473-482.

ZaJONC, R. (1980). Feeling and thinking: Preferences need no inferences. American Psychologist, 35, 151-175.

(Manuscript received for publication May 6, 1985.) 